\title{
High Amplitude Short Time Excitation: A Method to Form and Detect Low Mass Product Ions in a Quadrupole Ion Trap Mass Spectrometer
}

\author{
Connell Cunningham, Jr. and Gary L. Glish \\ Department of Chemistry, University of North Carolina at Chapel Hill, Chapel Hill, North Carolina, USA
}

\author{
David J. Burinsky \\ GlaxoSmithKline, Research Triangle Park, North Carolina, USA
}

Collision induced dissociation (CID) in a quadrupole ion trap mass spectrometer using the conventional $30 \mathrm{~ms}$ activation time is compared with high amplitude short time excitation (HASTE) CID using $2 \mathrm{~ms}$ and $1 \mathrm{~ms}$ activation times. As a result of the shorter activation times, dissociation of the parent ions using the HASTE CID technique requires resonance excitation voltages greater than conventional CID. After activation, the rf trapping voltage is lowered to allow product ions below the low mass cut-off to be trapped. The HASTE CID spectra are notably different from those obtained using conventional CID and can include product ions below the low mass cut-off for the parent ions of interest. The MS/MS efficiencies of HASTE CID are not significantly different when compared with the conventional $30 \mathrm{~ms}$ CID. Similar results were obtained with a two-dimensional (linear) ion trap and a three-dimensional ion trap. (J Am Soc Mass Spectrom 2006, 17, 81-84) (c) 2005 American Society for Mass Spectrometry

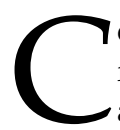
ollision induced dissociation (CID) remains by far the most common method used for ion activation in tandem mass spectrometry (MS/ MS) experiments [1, 2]. During CID, the internal energy of a parent ion is increased through inelastic collision(s) with a target gas, resulting in the dissociation of parent ions. CID is the result of two distinct events; parent ion activation (collisional activation) and parent ion dissociation [3]. The appearance of an MS/MS spectrum, both product ion formation and abundance, is a function of the amount of energy deposited into the parent ion during the activation period [4,5]. Activation times for CID in rf trapping instruments typically range between 10 and $50 \mathrm{~ms}$; however, CID can be accomplished using much shorter activation times in these instruments [6].

For $r f$ trapping instruments, the Mathieu parameters $q_{z}$ (proportional to the applied fundamental ac rf voltage), and $a_{z \prime}$ (proportional to the applied dc voltage) determine if ions of a given mass-to-charge will be trapped. Typically, and in these experiments, rf trapping instruments are operated at $a_{z}=0$, so theoretically

Published online December 15, 2005

Address reprint requests to Professor G. Glish, Campus Box 3290, Department of Chemistry, University of North Carolina at Chapel Hill, Chapel Hill, NC 27599-3290, USA. E-mail: glish@unc.edu ions with $q_{z}$ values from 0 to 0.908 are trapped. As the mass-to-charge value of an ion increases, its $q_{z}$ value decreases. Thus, the mass-to-charge value corresponding to a $q_{z}$ value of 0.908 is the minimum mass-to-charge value of ions trapped and is termed the low mass cut-off (LMCO).

Each ion held by the trapping field exists in a pseudo potential well with the well depth proportional to the $q_{z}$ for each mass-to-charge value. Hence, smaller mass-tocharge ions, which have larger $q_{z}$ values, exist in deeper wells than larger mass-to-charge ions, which have smaller $q_{z}$ values [7]. To implement CID, a $q_{z}$ value is selected that allows the kinetic energy of the parent ion to increase via power absorption from a supplementary resonance excitation voltage without exceeding the pseudo potential well depth. Typical $q_{z}$ values for CID in quadrupole ion traps range from 0.2 to 0.3 and supplementary resonance excitation voltages with amplitudes greater than $1 \mathrm{~V}$ are not generally utilized [8]. A trade-off in the selection of the $q_{z}$ value for CID is that product ions with mass-to-charge values below the LMCO will not be trapped. This means that product ions with mass-to-charge values less than $\sim 25-30 \%$ of the parent ion mass-to-charge are not observed in the MS/MS spectrum.

Previous research conducted using "fast excitation" CID has shown that collisional activation can be accom- 


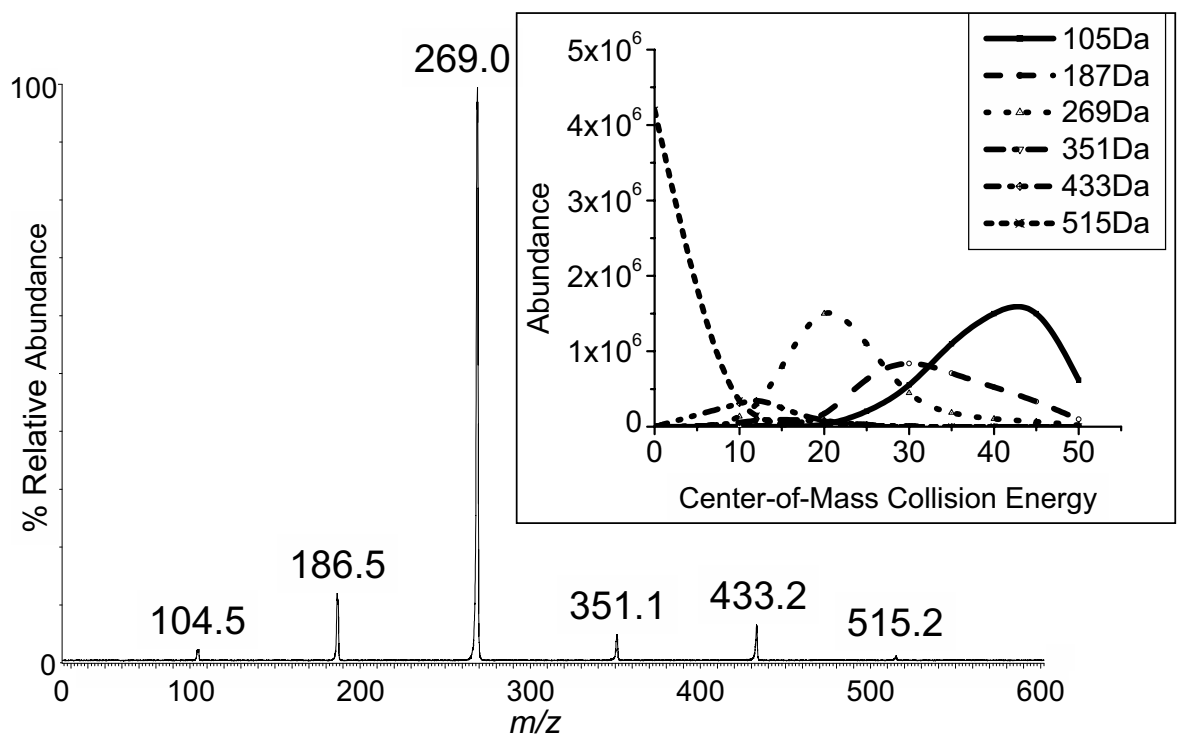

Figure 1. MS/MS spectra for $m / z 515$ sodiated sodium acetate parent ion using a QqQ at a collision energy of $20 \mathrm{eV}$. Inset: ERMS plot for CID of the $\mathrm{m} / \mathrm{z} 515$ parent ion using the QqQ.

plished in less than $100 \mu$ s in a quadrupole ion trap [6]. However, because a $19 \mathrm{~V}$ supplementary AC voltage was used, a $q_{z}$ value of 0.4 was required to reduce the number of parent ions being ejected from the trap. These previous experiments produced MS/MS spectra that were similar to those obtained using conventional activation times (30 ms), and MS/MS (CID) efficiencies decreased by about a factor of two compared with conventional activation times.

The research reported herein describes the results of excitation using high amplitude short time excitation (HASTE) CID with 1 and $2 \mathrm{~ms}$ activation times at the more conventional $q_{z}$ value of 0.25 . Both a ThermoElectron (San Jose, CA) (LTQ) linear ion trap mass spectrometer and a Finnigan 3D ion trap mass spectrometer (ITMS) were used. The results were compared with those obtained with a Waters Micromass (Manchester, UK) triple quadrupole mass spectrometer (QqQ). Spectra from both ion trap mass analyzers contain product ions below the LMCO defined by the parent ions' $q_{z}$ value. In addition, the abundance of product ions in the lower mass-to-charge range, but above the LMCO, increased as a result of the HASTE CID process.

CID performed in a triple quadrupole mass spectrometer is the result of multiple collisions of the parent ions with a collision gas which is typically argon. Parent ions are selected using the first quadrupole and then passed through a second multipole which serves as the collision region. The resulting product ions are mass analyzed using the third quadrupole $[8,9]$. Product ions that are formed near the beginning of the collision region can undergo multiple collisions and dissociate into additional generations of product ions, although from this single experiment it is not possible to determine if the product ions are formed by competitive or consecutive dissociation pathways.
Figure 1 shows the QqQ results for CID of a sodiated sodium acetate ion $\left(\mathrm{C}_{2} \mathrm{H}_{3} \mathrm{O}_{2} \mathrm{Na}\right)_{6} \mathrm{Na}^{+}$of $\mathrm{m} / z 515$ at a collision energy of $20 \mathrm{eV}$. The $\mathrm{m} / \mathrm{z} 105$ product ion is the lowest mass-to-charge ion observed in Figure 1 and represents the single sodiated sodium acetate ion, which, of all product ions, requires the most internal energy to form. The inset in Figure 1 is the energy resolved mass spectrum (ERMS) plot for the $\mathrm{m} / \mathrm{z} 515$ parent ion. As the collision energy of the parent ion is increased, product ions are more likely to continue to dissociate to product ions of lower mass-to-charge. These results clearly show the consecutive dissociation process occurring to produce the product ions with lower mass-to-charge values. Results from ITMS double resonance experiments, during which the $\mathrm{m} / \mathrm{z} 433$ product ion was simultaneously ejected while the $\mathrm{m} / \mathrm{z} 515$ ion was dissociated, also show that a majority of product ions of $\mathrm{m} / \mathrm{z} 351,269,187$, and 105 come from the $\mathrm{m} / \mathrm{z}$ 433 product ion.

The QqQ, in contrast to $\mathrm{rf}$ trapping mass analyzers (LTQ or ITMS), has a LMCO that is not associated with the parent ion's $q_{z}$ value, but is instead a function of the fundamental $\mathrm{rf}$ drive amplitude applied to each of the collision multipoles. For this reason, the complete MS/MS spectrum from $m / z 50$ to 550 is obtained (Figure 1). However, because the LMCO in the trapping mass analyzers is dependent upon the parent ion's $q_{z}$ value, CID can result in a loss of possible product ions. For example, $m / z 142$ is the LMCO if a $q_{z}$ value of 0.25 is used to dissociate the $\mathrm{m} / \mathrm{z} 515$ sodiated sodium acetate pentamer parent ion. As a result of this LMCO the $\mathrm{m} / \mathrm{z}$ 105 product ion observed in the QqQ MS/MS spectrum will not be observed in the LTQ or ITMS MS/MS spectrum using the conventional CID approach.

CID is performed in rf trapping mass analyzers through the use of resonance excitation [2, 3, 10-12]. In 


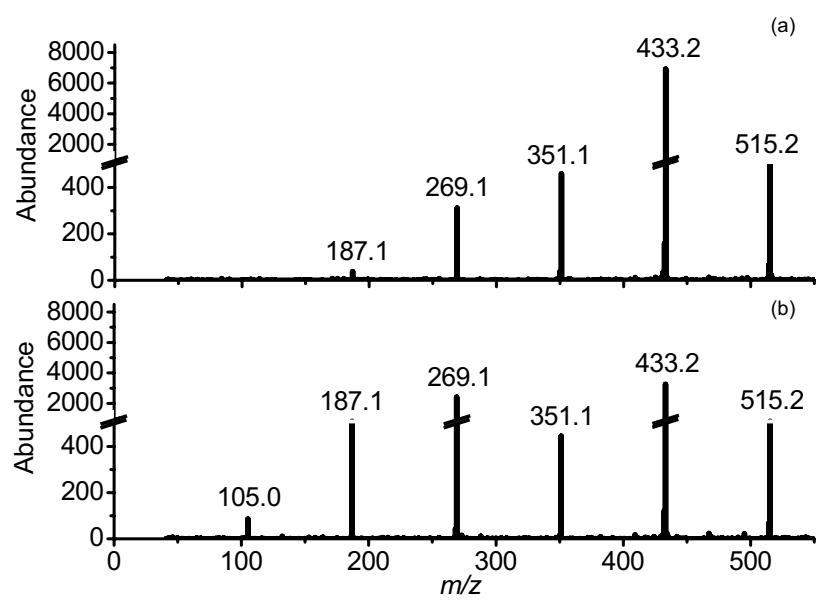

Figure 2. MS/MS spectra for $m / z 515$ from a 3D quadrupole ion trap mass spectrometer; (a) $30 \mathrm{~ms}$ activation time with a supplementary voltage of $165 \mathrm{mV}_{p-p}$, (b) 2 ms activation time with a supplementary voltage of $1025 \mathrm{mV}_{p-p}$.

the LTQ, CID is effected through the use of a supplementary ac voltage, with a frequency equal to the parent ion's secular frequency, applied in the radial direction [13]. For the ITMS, the supplementary voltage is applied in the axial direction. Any product ion that is formed as a result of CID is not in resonance with the applied supplementary voltage and, therefore, does not gain additional kinetic energy. Thus, only the parent ion gains kinetic energy during resonance excitation. Because ion activation is separate from dissociation, once a parent ion is activated, there is some time period before product ions are formed. Theoretically, if the trapping $\mathrm{rf}$ voltage is reduced quickly enough, product ions having mass-to-charge values below the LMCO during resonant excitation may be trapped for mass analysis.

Figure 2a shows the results of conventional $30 \mathrm{~ms}$ CID and Figure $2 b$ shows the results of 2 ms HASTE CID performed using the ITMS to dissociate the $m / z 515$ parent ion. Figure $2 \mathrm{a}$ is the result of a $165 \mathrm{mV}_{p-p}$ supplementary voltage and Figure $2 \mathrm{~b}$ is the result of $\mathrm{a}$ $1025 \mathrm{mV}_{p-p}$ supplementary voltage. A $1 \mathrm{~ms}$ activation time was also used to dissociate the $m / z 515$ parent ion (not shown) with a supplementary voltage of 4205 $\mathrm{mV}_{p-p}$ to produce a spectrum similar to the $2 \mathrm{~ms} / 1025$ $\mathrm{mV}_{p-p}$ MS/MS spectrum.

Following both the $2 \mathrm{~ms}$ and $1 \mathrm{~ms}$ activation times, the fundamental rf voltage was ramped down in $0.1 \mathrm{~ms}$ to a value resulting in a LMCO of $m / z$ 40. In Figure 2a, the $\mathrm{m} / \mathrm{z} 433$ product ion is clearly the most abundant peak in the spectrum. Because the $\mathrm{m} / \mathrm{z} 433$ product ion is not in resonance with the applied supplementary voltage, it does not gain any kinetic energy after it is formed. Instead, this ion begins to cool through collisions with the bath gas. However, in Figure $2 \mathbf{b}$ the $\mathrm{m} / \mathrm{z}$ 433 product ion displays an abundance of about $50 \%$ of that observed in Figure 2a. In addition, the $m / z$ 105, 187, and 269 product ions have each increased in abundance by at least $400 \%$ in the 2 ms HASTE CID spectrum. These results indicate that more internal energy is added to the parent ion during the 2 ms HASTE CID experiments than during the conventional $30 \mathrm{~ms}$ CID experiments. In addition, with HASTE CID the product ions below the LMCO are observed if the fundamental rf voltage is quickly ramped down following excitation.

In contrast to the software used to control the ITMS, which allows the user complete control over the voltages and times used in the experiment, the software that controls the LTQ provides limited user control and thus required an unconventional mode of operation to observe product ions below the LMCO when dissociating the $m / z 515$ parent ion. A "pseudo MS" acquisition was performed to determine if the ions below the LMCO could be observed using either conventional CID or HASTE CID. The parent ion was first dissociated using the conditions for conventional $30 \mathrm{~ms}$ CID and 2 ms HASTE CID. During the "MS" segment of the experiment, an activation mass of $m / z 140$ was specified along with an isolation window of one-hundred (the maximum isolation width allowed by the software) and a collision energy of zero. This procedure forced the LTQ to start scanning at $m / z 50$ instead of $m / z$ 140, the latter being the LMCO during the dissociation of the $\mathrm{m} / \mathrm{z} 515$ parent ion.

Figure 3 shows the results of this experiment using the LTQ instrument. The spectrum in Figure $3 \mathrm{a}$ is the result of a $30 \mathrm{~ms}$ activation time using 13\% normalized collision energy $\left(0.449 \mathrm{~V}_{p-p}\right)$ [14]. The most abundant product ion in Figure $3 a$ is the $\mathrm{m} / \mathrm{z} 433$ product ion. Figure $3 \mathrm{~b}$ is the result of a $2 \mathrm{~ms}$ activation time using HASTE CID with 29\% normalized collision energy $\left(1.035 \mathrm{~V}_{p-p}\right)$. This HASTE spectrum shows the $m / z 269$ ion as the most abundant product ion and the $\mathrm{m} / \mathrm{z} 105$ product ion exhibiting a signal 16 fold greater than that shown in Figure 3a.

There are two factors contributing to the different results observed between the $30 \mathrm{~ms}$ conventional CID and 2 ms HASTE CID spectra. First, the internal energy
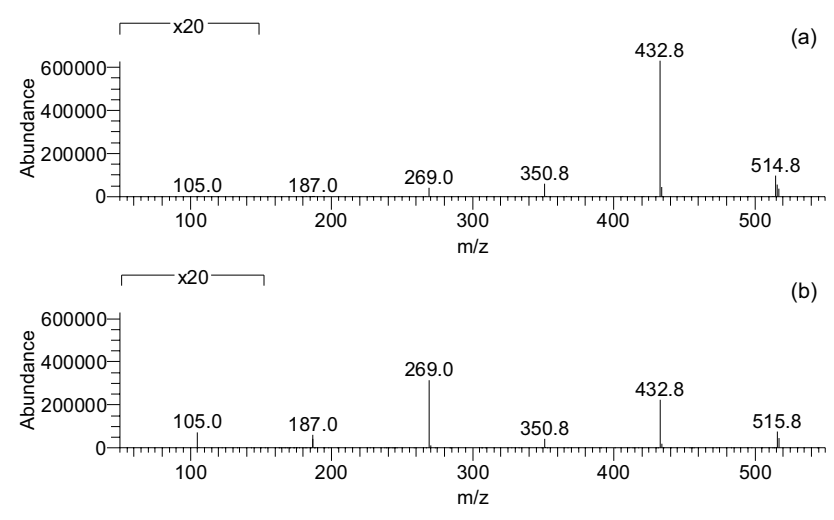

Figure 3. MS/MS spectra for $m / z 515$ from a 2D linear ion trap using "pseudo $\mathrm{MS}^{3}$ " technique to observe ions below low mass cut off; (a) $30 \mathrm{~ms}$ activation time with 13\% normalized collision energy, (b) $2 \mathrm{~ms}$ activation time with $29 \%$ normalized collision energy. 
Table 1. CID efficiencies for dissociation of the $m / z 515$ sodiated sodium acetate ion

\begin{tabular}{ccc}
\hline $\begin{array}{l}\text { Activation } \\
\text { time }(\mathrm{ms})\end{array}$ & $\begin{array}{c}\text { ITMS CID } \\
\text { efficiency }\end{array}$ & $\begin{array}{c}\text { LTQ CID } \\
\text { efficiency }\end{array}$ \\
\hline \hline 30 & $89.3 \%$ & $48.5 \%$ \\
2 & $74.5 \%$ & $41.4 \%$ \\
1 & $68.6 \%$ & - \\
\hline
\end{tabular}

gained by the parent ion before it dissociates is less for the $30 \mathrm{~ms}$ activation time compared to the $2 \mathrm{~ms}$ activation time; therefore, less of the $\mathrm{m} / \mathrm{z} 105$ product ion is likely to be produced. The second factor is that any $\mathrm{m} / \mathrm{z}$ 105 product ion formed during the $30 \mathrm{~ms}$ activation time period will likely be ejected out of the trap before the trapping voltage is lowered and the restoring force applied to the ion.

One of the most important features of quadrupole ion trap instruments is their high CID (MS/MS) efficiency. Table 1 shows the CID efficiency for all experiments, where CID

$$
E_{C I D}=\frac{\sum F}{P_{o}}
$$

efficiency is defined in eq 1 as the sum of the ion signal intensities of product ions, $F$, divided by the initial ion signal intensity of the parent ion, $P_{o}$. The CID efficiency for the LTQ instrument is lower than the ITMS, but does not vary greatly as a function of the activation time. This result suggests that the number of product ions produced during the $30 \mathrm{~ms}$ activation time is similar to the number of product ions produced during the $2 \mathrm{~ms}$ HASTE CID. The ITMS has the highest CID efficiency for the $30 \mathrm{~ms}$ activation time experiment, with the efficiency decreasing somewhat with shorter activation times. This decreased efficiency is most likely a result of ejecting some portion of the parent ions before they dissociate due to the increased supplementary voltage required to activate the parent ion at the shorter activation times.

Compared with conventional CID in a quadrupole ion trap, HASTE CID increases the internal energy of parent ions during collisional activation, resulting in more extensive consecutive dissociation processes and, consequently, an increased number of product ions at lower mass-to-charge values. A rapid decrease in the trapping voltage immediately after resonant excitation enables the detection of product ions with mass-tocharge values that fall below the typical LMCO imposed by the amplitude of the rf drive potential applied during CID. MS/MS spectra similar to those observed with a triple quadrupole mass spectrometer may be obtained. Preliminary results using peptides indicate that structurally diagnostic immonium ions can be formed and detected. Theoretical modeling in our laboratory using peptide ions shows an increase in internal temperature relative to conventional CID of the parent ions as a result of the HASTE CID process. These results are obtained with some loss of CID efficiency due to loss of parent ions during resonant excitation. However, the CID efficiency stills exceeds that obtained with other MS/MS instruments.

\section{Acknowledgments}

CC, Jr. acknowledges the Research Education Support Program at the University of North Carolina at Chapel Hill and the Alliance for Graduate Education and the Professoriate (NSF HRD 9978874) for partial support during this project.

\section{References}

1. Plass, W. R.; Cooks, R. G. A Model for Energy Transfer in Inelastic Molecular Collisions Applicable at Steady State or Nonsteady State and for an Arbitrary Distribution of Collision Energies. J. Am. Soc. Mass Spectrom. 2003, 14, 1348-1359.

2. McLuckey, S. A. Principles of Collisional Activation in Analytical Mass Spectrometry. J. Am. Soc. Mass Spectrom. 1992, 3, 599-614.

3. Busch, K. L.; Glish, G. L. McLuckey, S. A. Mass Spectrometry/Mass Spectrometry: Techniques and Applications of Tandem Mass Spectrometry; VCH: New York, 1988.

4. Vekey, K. Internal Energy Effects in Mass Spectrometry. I. Mass Spectrom. 1996, 31, 445-463.

5. McLuckey, S. A.; Goeringer, D. E. Slow Heating Methods in Tandem Mass Spectrometry. J. Mass Spectrom. 1997, 32, 461-474.

6. Murrell, J.; Despeyroux, D.; Lammert, S. A.; Stephenson, J. L.; Goeringer, D. E. "Fast Excitation" CID in a Quadrupole Ion Trap Mass Spectrometer. J. Am. Soc. Mass Spectrom. 2003, 14, 785-789.

7. March, R. E.; Todd, J. F. J. Practical Aspects of Ion Trap Mass Spectrometry, Vol I-III; CRC Press: New York, 1997.

8. Johnson, J. V. Yost, R. A.; Kelley, P. E.; Bradford, D. C. Tandem-inSpace and Tandem-in-Time Mass Spectrometry: Triple Quadrupoles and Quadrupole Ion Traps. Anal. Chem. 1990, 62, 2162-2172.

9. Hager, J. W. Recent Trends in Mass Spectrometer Development. Anal. Bioanal. Chem. 2004, 378, 845-850.

10. Konishi, Y.; Feng, R. Conformational Stability of Heme Proteins in Vacuo. Biochemistry 1994, 33, 9706-9711.

11. March, R. E. An Introduction to Quadrupole Ion Trap Mass Spectrometry. J. Mass Spectrom. 1997, 32, 351-369.

12. Sleno, L.; Volmer, D. A. Ion Activation Methods for Tandem Mass Spectrometry. J. Mass Spectrom. 2004, 39, 1091-1112.

13. Schwartz, J. C.; Senko, M. W.; Syka, J. E. P. A Two-Dimensional Quadrupole Ion Trap Mass Spectrometer. J. Am. Soc. Mass Spectrom. 2002, 13, 659-669.

14. Lopez, L. L.; Tiller, P. R.; Senko, M. W.; Schwartz, J. C. Automated Strategies for Obtaining Standardized Collisionally Induced Dissociation Spectra on a Benchtop Ion Trap Mass Spectrometer. Rapid Commun. Mass Spectrom. 1999, 13, 663-668. 